Citation: Yassine, K . "Weak solutions of first-order differential inclusions in Banach space". Journal of Engineering Technology and Applied Sciences 1 (1) $2016: 1-11$

\title{
WEAK SOLUTIONS OF FIRST-ORDER DIFFERENTIAL INCLUSIONS IN BANACH SPACE
}

\author{
Yassine Khouni \\ Faculty of Life and Natural Science, University of Batna, Algeria \\ yacinespoire@yahoo.fr
}

\begin{abstract}
The aim of this paper is to investigate the existence of pseudo-solutions for a First- order multivalued differential equation with nonlocal integral boundary condition in a Banach space.

Our approach is based on the use of the technique of measures of weak noncompactness and a fixedpoint theorem of Mönch type.
\end{abstract}

Keywords:Pseudo-solutions, differential inclusions, integral boundary condition, measure of weak noncompactness, pettis integral.

\section{Introduction}

There are many problems in applied mathematics such as: control theory, economical systems, Hamiltonian system, that lead us to the study of differential inclusions

$$
x^{\prime}(t) \in F(t, x(t))
$$

where $F(.,$.$) is a set valued map (see [3] and [11] for instance and references there in).$

The main purpose of this paper is to establish the existence of pseudo-solutions to the nonlocal boundary value problems of integral type

$$
\left\{\begin{array}{l}
x^{\prime}(t) \in F(t, x(t)), \quad t \in I=[0, T] \\
x(0)+\mu \int_{0}^{T} x(s) d s=x(T)
\end{array}\right.
$$

where $F: I \times E \rightarrow P(E)$ is a multivalued map, $E$ is a Banach space with the norm $\|$.$\| ,$ $P(E)$ is the family of all subsets of $E$ and $\mu \in \mathbb{R}^{\star}$ 
Nonlocal problems for ordinary differential equations (single valued $F$ ) have been investigated by several authors (see for instance [12] and references therein), also classical initial values problems for multivaled differential equations have been considered by many authors (see [15]) and nonlocal differential inclusions have been studied by many authors (see for instance [9] and the references therein).

Boundary value problems with integral boundary conditions constitute a very interesting and important class of problems. They include two, three, multi-point and nonlocal boundary value problems as special cases. Integral boundary conditions are often encountered in various applications, it is worthwhile mentioning the applications of those conditions in the study of population dynamics [8] and cellular systems [1]. Moreover, boundary value problems with integral boundary conditions have been studied by a number of authors such as Arara and Benchohra [2], Benchohra et al [5 ]- [7], Infante [14] and references therein.

In our investigation, we apply the method associated with the technique of measures of weak noncompactness and fixed point theorem of Mönch type.

The remainder of this paper is organized as follows. In section 2, we present some basic definitions and notations about pseudo-solutions and multivalued map. In section 3, we give main results for nonlocal boundary value problem for differential inclusions of integral type.

\section{Preliminaries}

In this section, we introduce notations, definitions and preliminary facts that used in the remainder of this paper. Let $E$ be a real Banach space with the norm $\|$.$\| and dual space E^{\star}$, let $C(I ; E)$ be the Banach space of all continuous functions from $I$ to $E$ with the norm

$$
\|y\|_{\infty}=\sup \{\|y(t)\| ; \quad 0 \leq t \leq T\}
$$

and let $L^{1}(I ; E)$ denote the Banach space of functions $y: I \rightarrow E$ that are lebesgue integrable with norm

$$
\|y\|_{L^{1}}=\int_{0}^{T}\|y(t)\| d t
$$

let $L^{\infty}(I, E)$ to be the Banach space of bounded measurable functions $y: I \rightarrow E$ equipped with the norm

$$
\|y\|_{L^{\infty}}=\inf \{c>0 ;\|y(t)\| \leq c, \text { a.e.t } \in I\}
$$

Also let $P(E)$ is the set of all nonempty subsets of $E$.

$P_{c l}(E)=\{Y \in P(E) ; \quad Y$ is closed $\}$.

$P_{c v}(E)=\{Y \in P(E) ; \quad Y$ is convex $\}$.

$P_{b d}(E)=\{Y \in P(E) ; \quad Y$ is bounded $\}$.

$P_{c l, c v}(E)=\{Y \in P(E) ; \quad Y$ is closed and convex $\}$. 
$P_{c p, c v}(E)=\{Y \in P(E) ; \quad Y$ is compact and convex $\}$.

Definition 2.1: A set-valued function $F: E \rightarrow P(E)$ is called convex, closed and compact valued respectively if $F(x)$ is convex, closed, compact respectively for all $x \in E$.

Definition 2.2: A set-valued function $F: E \rightarrow P(E)$ is called bounded valued on bounded sets $B$ if $F(B)=\bigcup_{x \in B} F(x)$ is bounded in $E$ for all $B \in P_{b d}(E)$ or equivalently;

$$
\sup _{x \in B}\{\sup \{|u| ; u \in F(x)\}\}<\infty \text {. }
$$

Definition 2.3: A set-valued function $F: E \rightarrow P(E)$ is called upper semicontinuous (u.s.c) on $E$ if for each $x_{0} \in E$ the set $F\left(x_{0}\right)$ is nonempty closed subset of $E$ and if for each open set $N$ containing $F\left(x_{0}\right)$, there exist an open neighborhood $N_{0}$ of $x_{0}$ such that $F\left(N_{0}\right) \subseteq N$. In other words, $F$ is u.s.c if the set $F^{-1}(A)=\{x \in E: F(x) \in A\}$ is open in $E$ for every open set $A$ in $E$. Or if every closed subset $A$ of $E$ the set $F^{+}(A)=\{x \in E: \quad A \cap F(x) \neq \emptyset\}$ is closed in $E$.

Definition 2.4: A set-valued function $F: E \rightarrow P(E)$ is called sequentially weakly upper semicontinuous (w.u.s.c) if $F$ is u.s.c with respect to the weak topology of $E$.

Definition 2.5: A set-valued function $F: I \rightarrow P(E)$ is said to be measurable if for any $t \in I$, the function $t \mapsto d(x, F(t))=\inf \{|x-u|: \quad u \in F(t)\}$ is measurable. Or for every closed set $M, F^{-1}(M)$ is measurable.

Definition 2.6: $x():. I \rightarrow E$ is called weakly continuous (measurable) at $t_{0} \in I$ if for every $\varphi \in$ $E^{\star}, \varphi(x()$.$) is continuous (measurable) at t_{0}$.

Definition 2.7: A family $G=\left\{f_{i}, i \in J(\right.$ index $\left.)\right\}$ is said to be weakly equicontinuous if given $\varepsilon>0, \varphi \in E^{\star}$ there exists $\delta>0$ such that for each $t, s \in I$ if $|t-s|<\delta$ then $\left|\varphi\left(f_{i}(t)-f_{i}(s)\right)\right|<\varepsilon$ for all $i \in J$.

Definition 2.8: The function $x():. I \rightarrow E$ is said to be Pettis integrable on $I$ if and only if there is an element $x_{J} \in E$ corresponding to each $J \subseteq I$ such that $\varphi\left(x_{J}\right)=\int_{J} \varphi(x(s)) d s$ for all $\varphi \in$ $E^{\star}$ where the integral in the right is assumed to exist in the sense of Lebesgue. By definition

$$
x_{J}=\int_{J} x(s) d s
$$

We denote $P^{1}(I)$ the space of Pettis integrable functions on $I$.

$S_{F}(x)=\left\{f \in P^{1}(I): f \in F\right\}$ denotes the set of Pettis selections functions of $F$, it is clear that

$$
S_{F}(x) \supset S_{F, x}=\left\{f \in L^{1}(I): f \in F\right\} .
$$

Definition 2.9: Let $\Omega_{E}$ be the family of bounded subsets of $E$ and $B_{1}$ be the unit ball in $E$. The De Blasi measure of weak noncompactness is the map

defined by

$$
\beta: \Omega_{E} \rightarrow[0,+\infty]
$$




$$
\beta(X)=\inf \left\{\varepsilon>0: \quad \text { there exists a weakly compact subset } \Omega \text { of } E \text { such that } X \subset \Omega+\varepsilon B_{1}\right\}
$$

Lemma 2.10: Let $A, B$ be bounded subsets of $E$ and $\left\{x_{n}\right\},\left\{y_{n}\right\}$ be bounded sequences in $E$. Then:

1- $A \subseteq B$ then $\beta(A) \leq \beta(B)$,

2- $\beta(A)=\beta\left(\overline{A^{w}}\right)$ where $\overline{A^{w}}$ denotes the weak closure of $A$,

3- $\beta(A)=0$ if and only if $\overline{A^{w}}$ is weakly compact,

4- $\beta(A \cup B)=\max (\beta(A), \beta(B))$,

5- $\beta(A)=\beta(\operatorname{Co}(A))$,

6- $\beta(A+B) \leq \beta(A)+\beta(B)$,

7- $\beta\left(\left\{x_{n}\right\}\right)-\beta\left(\left\{y_{n}\right\}\right) \leq \beta\left(\left\{x_{n}-y_{n}\right\}\right)$,

8- $\beta(x+A)=\beta(A)$ where $x \in E$,

9- $\beta(t A)=t \beta(A), t \geq 0$.

Proof. Proof See [10]

Proposition 1: Let $E$ be a normed space and element $x_{0} \neq 0$. Then there exists $\varphi \in E^{\star}$ with $\|\varphi\|=1$ and $\varphi\left(x_{0}\right)=\left\|x_{0}\right\|$.

Proof. See [19] (Chapter IV, Corollary 2).

Definition 2.11: A function $x(.) \in C(I, E)$ is said to be weakly differentiable if $\varphi(x()$.$) is$ derivable for every $\varphi \in E^{\star}$.

Definition 2.12: A function $x(.) \in C(I, E)$ is said to be pseudo-differentiable on $I$ to a function

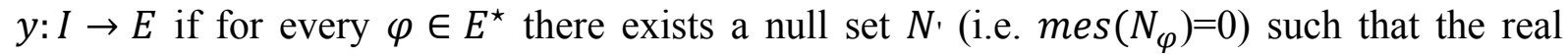
function $t \mapsto(\varphi, x(t))$ is differentiable on $I \backslash N_{\varphi^{\prime}}$ and

$$
\frac{d}{d t}(\varphi, x(t))=(\varphi, y(t)), \quad t \in I \backslash N_{\varphi},
$$

The function $y$ is called a pseudo-derivative of $x$ and it will denoted by $x^{\prime}($.$) or by \frac{d_{p}}{d t} x($.$) .$

In the other words $(\varphi, x()$.$) differentiable a.e. on I$.

Definition 2.13: A function $x():. I \rightarrow E$ is said to be a pseudo-solution of problem (1)-(2) if it satisfies the following conditions

$1-x($.$) is absolutely continuous,$ 
2- $x(0)+\mu \int_{0}^{T} x(s) d s=x(T)$,

3- For each $\varphi \in E^{\star}$ there exists a null set $N_{\varphi}$ such that for each $t \in I \backslash N_{\varphi}$,

$$
\varphi\left(x^{\prime}(t)\right)=\varphi(v(t))
$$

Where $v(t) \in F(t, x(t)), t \in I$ and ' denotes a pseudo-derivative.

In other words by a pseudo-solution of the problem (1) - (2) we will understand an absolutely continuous function $x($.$) such that x(0)+\mu \int_{0}^{T} x(s) d s=x(T)$ and for each $\varphi \in E^{\star}, x($. satisfies the following:

$$
\varphi\left(x^{\prime}(t)\right)=\varphi(v(t)), \text { a.e. } t \in I
$$

where $v(t) \in F(t, x(t)), t \in I$.

Remark 2.14: If $g$ is Pettis integrable and $x(t)=\int_{0}^{t} g(s) d s$, then $x($.$) is weakly differentiable$ and $x^{\prime}(t)=g(t)$.

If $x():. I \rightarrow E$ is a function weakly differentiable on $I$, then we have

$$
\frac{d}{d t}(\varphi, x(t))=\left(\varphi, x^{\prime}(t)\right), \quad t \in I
$$

for every $\varphi \in E^{\star}$.

Definition 2.15: A function $F: E \rightarrow P_{c l, c v}(E)$ has a weakly sequentially closed graph if for any sequence $\left(x_{n}, y_{n}\right) \in E \times E, y_{n} \in F\left(x_{n}\right)$ for $n \in\{0,1,2,3, \ldots\}$ with $x_{n}(t) \rightarrow x(t)$ for each $t \in I$ and $y_{n}(t) \rightarrow y(t)$ for each $t \in I$, then $y \in F(x)$, where $\rightarrow$ denote a weak convergence.

Lemma 2.16: If $x($.$) is Pettis integrable and h($.$) is a measurable and essentially bounded real$ valued function, then $x() h.($.$) is Pettis integrable.$

Next, we shall use the following fundamental theorem

Theorem 2.17: (Mönch fixed point theorem) Let $E$ be a Banach space with $Q$ a nonempty, bounded, closed, convex, equicontinuous subset of $C(I, E)$.

Suppose $N: Q \rightarrow P_{c l, c v}(Q)$ has a weakly sequentially closed graph. If the implication

$$
\bar{V}=\overline{c o n v}(N(V) \cup\{0\}) \Rightarrow V \text { is relativelly weakly compact }
$$

holds for every subset $V$ of $Q$, then the operator inclusion $x \in N(x)$ has a solution in $Q$. 


\section{The main result}

In this section we give the state and the proof of our result.

Firstly we have the following lemma

Lemma 3.1: Let $h(.) \in P^{1}(I)$ be a given function, then the boundary value problem

$$
\left\{\begin{array}{l}
x^{\prime}(t)=h(t), \quad t \in I=[0, T] \\
x(0)+\mu \int_{0}^{T} x(s) d s=x(T)
\end{array}\right.
$$

has a solution given by

$$
x(t)=\int_{0}^{T} G(t, s) h(s) d s
$$

where $G(.,$.$) is the function defined by the formula:$

$$
G(t, s)= \begin{cases}\frac{s}{T}+\frac{1}{\mu T}, & 0 \leq s<t \\ \frac{-(T-s)}{T}+\frac{1}{\mu T}, & t \leq s \leq T\end{cases}
$$

Proof: we can reduce the equation (3) to an equivalent integral equation

$$
x(t)=x(0)+\int_{0}^{t} h(s) d s
$$

By integration we have (using Fubini's integral theorem)

$$
\begin{gathered}
\int_{0}^{T} x(s) d s=\int_{0}^{T} x(0) d s+\int_{0}^{T}\left(\int_{0}^{s} h(\tau) d \tau\right) d s=T x(0)+\int_{0}^{T}\left(\int_{\tau}^{T} d s\right) h(\tau) d \tau \\
=\operatorname{Tx}(0)+\int_{0}^{T}(T-\tau) h(\tau) d \tau
\end{gathered}
$$

applying the boundary condition (4), we get:

$$
\mu \int_{0}^{T} x(s) d s=\mu T x(0)+\mu \int_{0}^{T}(T-\tau) h(\tau) d \tau
$$

this implies that

$$
x(T)-x(0)=\mu T x(0)+\mu \int_{0}^{T}(T-\tau) h(\tau) d \tau
$$


hence

$$
x(0)+\int_{0}^{T} h(s) d s-x(0)=\mu T x(0)+\mu \int_{0}^{T}(T-\tau) h(\tau) d \tau
$$

therefore

$$
x(0)=\frac{1}{\mu T}\left[\int_{0}^{T} h(s) d s-\mu \int_{0}^{T}(T-s) h(s) d s\right]
$$

hence

$$
\begin{aligned}
x(t)=\frac{1}{\mu T}\left[\int_{0}^{T}\right. & \left.h(s) d s-\mu \int_{0}^{T}(T-s) h(s) d s\right]+\int_{0}^{t} h(s) d s \\
& =\frac{1}{\mu T} \int_{0}^{t}(1-\mu(T-s)) h(s) d s+\frac{1}{\mu T} \int_{t}^{T}(1-\mu(T-s)) h(s) d s+\int_{0}^{t} h(s) d s
\end{aligned}
$$

This implies that

$$
x(t)=\int_{0}^{T} G(t, s) h(s) d s
$$

which completes the proof.

Remark 3.2: The function $t \mapsto \int_{0}^{T}|G(t, s)| d s$ is continuous on $I$, and hence is bounded, let

$$
\tilde{G}=\sup \left\{\int_{0}^{T}|G(t, s)| d s ; \quad t \in I\right\} .
$$

Now, we are in position to state and prove our existence result for the problem (1)-(2), firstly we need the following assumptions;

a) The set-valued function $F: I \times E \rightarrow P_{c p, c v}(E)$ is measurable in the first variable and $x \mapsto$ $F(t, x)$ is sequentially weakly upper semicontinuous a.e. $t \in I$,

b) There exist $\varphi_{F} \in L^{\infty}\left(I, \mathbb{R}_{+}\right)$and a continuous nondecreasing function $\rho_{F}:[0,+\infty[\rightarrow$ $[0,+\infty[$ such that

$$
\|F(t, x)\|=\sup \{|v| ; \quad v \in F(t, x)\} \leq \varphi_{F}(t) \rho_{F}(\|x\|),
$$

c) There exists a constant $R>0$ such that

$$
\frac{R}{\tilde{G}\left\|\varphi_{F}\right\|_{L^{\infty} \rho_{F}(R)}}>1
$$

d) For each bounded set $Q \subset E$ and each $t \in I$, 


$$
\beta(F(t, Q)) \leq \varphi_{F}(t) \beta(Q) .
$$

Theorem 3.3: Assume that the assumptions a)-b) are satisfied. If

$$
\left\|\varphi_{F}\right\|_{L^{\infty}} \tilde{G}<1,
$$

then, the boundary value problem (1) - (2) has at least one solution.

Proof: From the assumptions a)-b) we deduce that there exists a petties selection function $f: I \rightarrow E$ of $F$ (i.e. $f(t) \in F(t, x(t)), \forall t \in I)$.

Now, we transform the problem (1) - (2) into fixed point problem by considering the multivalued operator $N: C(I, E) \rightarrow P_{c l, c v}(C(I, E))$ defined by

$$
N(x)=\left\{h \in C(I, E) ; \quad h(t)=\int_{0}^{T} G(t, s) f(s) d s, f \in S_{F}(x)\right\},
$$

Firstly, we show that the operator $N$ makes sense, to see this, let $x \in C(I, E)$, by a)-b) there exists a pettis integrable function $f: I \rightarrow E$ such that $f(t) \in F(t, x(t))$ for a.e. $t \in I$.

Since $G(t,.) \in L^{\infty}$, then $G(t,) f.($.$) is pettis integrable and thus N$ is well defined.

Let $R>0$, and consider the set

$$
\begin{aligned}
& Q=\left\{x \in C(I, E) ; \quad\|x\|_{\infty} \leq R \text { and }\left\|x\left(t_{1}\right)-x\left(t_{2}\right)\right\|\right. \\
& \left.\quad \leq\left\|\varphi_{F}\right\|_{L^{\infty}} \rho_{F}(R) \int_{0}^{T}\left|G\left(t_{1}, s\right)-G\left(t_{2}, s\right)\right| d s \text { for } t_{1}, t_{2} \in I\right\} .
\end{aligned}
$$

Notice that $Q$ is a closed, convex, bounded and equicontinuous subset of $C(I, E)$. We shall show that $N$ satisfies the assumptions of Mönch fixed point theorem, to see this we have several steps.

Step1: $N(x)$ is convex for each $x \in Q$.

Indeed, if $y_{1}$ and $y_{2}$ belong to $N(x)$, then there exists pettis integrable functions $f_{1}, f_{2}$ where $f_{1}(t), f_{2}(t) \in F(t, x(t))$ such that for all $t \in I$ we have

$$
y_{i}(t)=\int_{0}^{T} G(t, s) f_{i}(s) d s, \quad i=1,2 .
$$

Let $0 \leq \alpha \leq 1$, then, for each $t \in I$, we have

$$
\left(\alpha y_{1}+(1-\alpha) y_{2}\right)(t)=\int_{0}^{T} G(t, s)\left[\alpha f_{1}(s)+(1-\alpha) f_{2}(s)\right] d s,
$$

since $F$ has convex values, $\alpha f_{1}(t)+(1-\alpha) f_{2}(t) \in F(t, x(t))$, and we have $\left(\alpha y_{1}+(1-\alpha) y_{2}\right) \in N(x)$. 
Step 2: $N$ maps $Q$ into $Q$.

To see this, take $u \in N Q$, then there exists $x \in Q$ with $u \in N(x)$ and there exists a pettis integrable function $f: I \rightarrow E$ with $F(t, x(t))$ for a.e. $t \in I$, without loss of generality, we assume $u(s) \neq 0, \forall s \in I$, then there exists $\varphi \in E^{\star}$ with $\|\varphi\|=1$ and $\varphi(u(s))=\|u(s)\|$, hence, for each fixed $t \in I$, we have

$$
\|u(t)\|=\varphi(u(t))=\varphi\left(\int_{0}^{T} G(t, s) f(s) d s\right) \leq \int_{0}^{T}|G(t, s)| \varphi(f(s)) d s \leq \tilde{G}\left\|\varphi_{F}\right\|_{L^{\infty}} \rho_{F}\left(\|x\|_{\infty}\right)
$$

therefore

$$
\|u\|_{\infty}<R
$$

Now, suppose that $u \in N Q$ and $t_{1}, t_{2} \in I$ with $t_{1}<t_{2}$ so that $u\left(t_{2}\right)-u\left(t_{1}\right) \neq 0$, then, there exists $\varphi \in E^{\star}$ such that

$$
\begin{gathered}
\left\|u\left(t_{2}\right)-u\left(t_{1}\right)\right\|=\varphi\left(\int_{0}^{T}\left(G\left(t_{2}, s\right)-G\left(t_{1}, s\right)\right) f(s) d s\right) \\
\leq \int_{0}^{T}\left|G\left(t_{2}, s\right)-G\left(t_{1}, s\right)\right|\|f(s)\| d s \leq\left\|\varphi_{F}\right\|_{L^{\infty}} \rho_{F}(R) \int_{0}^{T}\left|G\left(t_{2}, s\right)-G\left(t_{1}, s\right)\right| d s
\end{gathered}
$$

therefore, $u \in Q$.

Step 3: $N$ has a weakly sequentially closed graph.

Let $\left(x_{n}, y_{n}\right)_{n}$ be a sequence in $Q \times Q$ with $x_{n}(t) \rightarrow x(t)$ for each $t \in I, y_{n}(t) \rightarrow y(t)$ for each $t \in I$, and $y_{n} \in N\left(x_{n}\right)$ for $n \in\{0,1,2, \ldots\}$. We shall show that $y \in N(x)$. By the relation $y_{n} \in N\left(x_{n}\right)$, we mean that there exists $f_{n} \in S_{F}\left(x_{n}\right)$ such that

$$
y_{n}(t)=\int_{0}^{T} G(t, s) f_{n}(s) d s
$$

we must show that there exists $f \in S_{F}(x)$ such that for each $t \in I$,

$$
y(t)=\int_{0}^{T} G(t, s) f(s) d s
$$

since $F$ has compact values (so weakly compact), then, there exists a subsequence $f_{n_{k}}$ such that

$$
f_{n_{k}}(t) \rightarrow f(t) \text { as } n_{k} \rightarrow 0
$$

and

$$
f_{n_{k}}(t) \in F\left(t, x_{n_{k}}(t)\right) \text { a.e. } t \in I
$$


we have also $F(t,$.$) has a weakly sequentially closed graph (because F(t,$.$) is sequentially$ weakly upper semicontinuous), $f(t) \in F(t, x(t))$ the Lebesque Dominated convergence theorem for pettis integral then implies that for each $\varphi \in E^{\star}$ we have

$$
\begin{gathered}
\varphi\left(y_{n}(\mathrm{t})\right)=\varphi\left(\int_{0}^{T} G(t, s) f_{n}(s) d s\right) \rightarrow \varphi\left(\int_{0}^{T} G(t, s) f(s) d s\right) \\
\text { i.e., } y_{n}(t) \rightarrow N(x)(t)
\end{gathered}
$$

we can repeat this for each $t \in I$, so $y(t) \in N(x)(t)$.

Step 4: The implication (*) holds.

Let $V$ be a subset of $Q$ such that $V=\overline{\operatorname{conv}}(N(V) \cup\{0\})$, clearly, $V(t) \subset \overline{\operatorname{conv}}(N V(t) \cup\{0\})$ for all $t \in I$. Also $N V(t) \subset N Q(t)$, for each $t \in I$, and is bounded in $P(E)$. By the properties of the measure $\beta$, we have

$$
\begin{gathered}
\beta(V(t)) \leq \beta(\overline{\operatorname{conv}}(N V(t) \cup\{0\}))=\beta(N V(t)) \\
=\beta\left(\int_{0}^{T} G(t, s) f(s) d s ; f(t) \in F(t, x(t)), \quad x \in V, t \in I\right) \\
\leq \int_{0}^{T}|G(t, s)| \varphi_{F}(s) \beta(V(s)) d s
\end{gathered}
$$

and therefore

$$
\|v\|_{\infty} \leq\|v\|_{\infty} \tilde{G} \int_{0}^{T} \varphi_{F}(s) d s
$$

where $\|v\|_{\infty}=\sup \{\beta(V(t)), \quad t \in I\}$.

This means that

$$
\|v\|_{\infty}\left[1-\left\|\varphi_{F}\right\|_{L^{\infty}} \bar{G}\right] \leq 0
$$

and hence $\|v\|_{\infty}=0$, thus, $V$ is weakly relatively compact. Applying Mönch fixed point theorem, we deduce that $N$ has a fixed point that is a solution of the problem (1) - (2).

\section{References}

[1] Adomian, G., G. E. Adomian, "Cellular Systems and Aging Models." Computers \& Mathematics with Applications 11.1-3 (1985): 283-291.

[2] Arara, A., M. Benchohra, "Fuzzy Solutions for Boundary Value Problems with Integral Boundary Conditions.” Acta Math. Univ. Comenianae 75.1 (2006): 119-126.

[3] Arino, O., S. Gautier, J. P. Penot, "A fixed point theorem for sequentially continuous mappings with application to ordinary differential equations." Funkcial. Ekvac 27.3 (1984): 273-279. 
[4] Aubin, Jean-Pierre, Arrigo Cellina, "Differential Inclusions with Maximal Monotone Maps. ” Differential Inclusions. Springer Berlin Heidelberg, 1984: 139-171.

[5] Benchohra, Mouffak, Samira Hamani, Johnny Henderson, "Functional Differential Inclusions with Integral Boundary Conditions." Electr. J. Qual. Theo. Differential Equations 15 (2007): 1-13.

[6] Benchohra, M., J. Graef, F. Z. Mostefai, "Weak Solutions for Boundary-Value Problems with Nonlinear Fractional Differential Inclusions." Nonlinear Dynamics and System Theory 11.3 (2011): 227-237.

[7] Benchohra, Mouffak, Fatima-Zohra Mostefai, "Weak Solutions for Nonlinear Fractional Differential Equations with Integral Boundary Conditions in Banach Spaces." Opuscula Mathematica 32.1 (2012): 31-40.

[8] Blayneh, K. W., “Analysis of Age Structured Host-Parasitoid Model.” Far East J. Dyn. Syst 4 (2002): 125-145.

[9] Cichoń, Kinga, Mieczysław Cichoń, Bianca Satco, "Differential Inclusions and Multivalued Integrals.” Discussiones Mathematicae, Differential Inclusions, Control and Optimization 33.2 (2013): 171-191.

[10] De Blasi, Francesco S., "On A Property of The Unit Sphere in A Banach Space." Bulletin Mathématique de la Société des Sciences Mathématiques de la République Socialiste de Roumanie (1977): 259-262.

[11] Górniewicz, Lech, Topological Fixed Point Theory of Multivalued Mappings. Vol. 495. Dordrecht: Kluwer Academic Publishers, 1999.

[12] Yassine, Khouni, "Weak Solutions of First-Order Differential Inclusions in Banach Space.” (2016): 1-10.

[13] R. W. Ibrahim, "The Existence of Weak Solutions for Fractional Integral Inclusions Involving Pettis Integral”. Journal of Scientific and Mathematical Research, (2008): 1-8.

[14] Infante, Gennaro, "Eigenvalues and Positive Solutions of Odes Involving Integral Boundary Conditions. ” Discrete Contin. Dyn. Syst 2005 (2005): 436-442.

[15] Wu Jianrong Xue Xiaoping Wu Congxin, "Existence theorem for weak solutions of Random differential inclusions in Banach spaces" Advences in Mathematics, 30, 4 (2001): 359-366.

[16] SPandey, S. K., "Existence of Measurable Selectors in Pettis Integrable Multi Function." Journal of Computer and Mathematical Sciences 5.1 (2014): 1-122.

[17] Pettis, Billy James, "On Integration in Vector Spaces." Transactions of the American Mathematical Society 44.2 (1938): 277-304.

[18] Vrabie, Ioan I., "Compactness Methods for Nonlinear Evolutions.” Vol. 75. CRC Press, 1995.

[19] Yosida, Kosaku, Functional Analysis. Vol. 6. Springer-Verlag. Berlin, 1980. 